\title{
PURIFICATION OF SEMICONDUCTING SINGLE-WALLED CARBON NANOTUBES BY SPIRAL
} \section{COUNTER-CURRENT CHROMATOGRAPHY}

\author{
${ }^{1}$ CC Biotech LLC, 12111 Parklawn Drive Rockville, MD 20852. ${ }^{2}$ Fuzbien Technology Institute, Inc., 12111
} Parklawn Drive Rockville, MD 20852.

*Corresponding author: Martha Knight, phone: 240-669-9281, marthaknight@starpower.net

\section{ABSTRACT}

Over the last decade man-made carbon nanostructures have shown great promise in electronic applications, but they are produced as very heterogeneous mixtures with different properties so the achievement of a significant commercial application has been elusive. The dimensions of single-wall carbon nanotubes are generally a nanometer wide, up to hundreds of microns long and the carbon nanotubes have anisotropic structures. They are processed to have shorter lengths but they need to be sorted by diameter and chirality. Thus counter-current chromatography methods developed for large molecules are applied to separate these compounds. A modified mixer-settler spiral CCC rotor made with $3 \mathrm{D}$ printed disks was used with a polyethylene glycol-dextran 2-phase solvent system and a surfactant gradient to purify the major species in a commercial preparation. We isolated the semiconducting single walled carbon nanotube chiral species identified by UV spectral analysis. The further development of spiral counter-current chromatography instrumentation and methods will enable the scalable purification of carbon nanotubes useful for the next generation electronics.

KEYWORDS carbon nanotubes, semiconductors, chiral types, mixer-settler rotors, gradient countercurrent chromatography (CCC), aqueous two-phase solvent system (ATPS) 


\subsection{Introduction}

24 The long awaited commercial application of single walled carbon nanotubes [1] depends on an

25 economically viable process to produce pure materials. Methods to seed and grow homogeneous

26 carbon nanotubes are being developed [2-3]. Nevertheless, the capability to produce chirally pure

27 carbon nanotube dispersions for uses such as printing on bio-sensor circuits will be greatly aided by scalable chromatographic separation. Carbon nanostructures with semiconductor properties provide very fast and sensitive electronic detection in advanced applications such as field-effect transistors, nano-scale sensors, conducting films, optimized near IR fluorophores and drug conjugates [4]. These technologies are developed for diagnostics and drug delivery. Since the processes of preparing chirally pure structures of single-walled carbon nanotubes (SWCNTS) are difficult and under intense development [5], it is worthwhile that methods proposed here can serve in separations of these macromolecules to highly homogeneous preparations. CC Biotech has been developing spiral CCC technologies specialized for large molecules. This includes designs of novel separation columns/rotors for use in a planet centrifuge with the aqueous two-phase solvent systems (ATPS) heretofore studied for protein purification [6]. An initial report of separating carbon nanotubes by high-speed CCC used an aq. organic solvent system, but no details on the types of carbon nanotubes isolated were provided [7]. More recently the utilization of ATPS in spiral CCC with the mixer-settler rotor served to achieve separation of chiral species [8], an important feat which opens up opportunities to develop more analytical and preparative methods to achieve the purification needed in the important applications.

42 Carbon nanotubes (CNTs) are carbon-carbon extended polymers in 2 dimensions with fused sp2 orbitals 43 having aromaticity [4-5]. The sheets form long tubes, the nanotubes; when they remain sheet-like they 44 are the graphenes. The tubular forms are of various diameters from less than $1 \mathrm{~nm}$ to a few hundred $45 \mathrm{~nm}$ and have lengths of $500 \mathrm{~nm}$ to several hundreds of $\mu \mathrm{m}$. Nanotubes are single-walled, double-walled 46 or multi-walled, having anisotropic structures. They exhibit conductive or semi-conductor properties 
47 depending on their chirality. The hexagonal array of the atoms in the polymeric tubes have a left-

48 handed or right-handed spiral pattern. The dimensions of the patterns of the hexagonal honeycomb-

49 like lattice are described by vectors, with certain values of $m, n$ being semi-conducting. The pattern 'zig-

50 zag' where $\mathrm{m}=0$ and 'arm chair' where $\mathrm{n}=\mathrm{m}$ (metallic ones) are the non-chiral structures. More

51 specifically, chirality of SWCNTs are completely described by a single vector C (chiral vector). Two atoms

52 in a planar graphene sheet are chosen and one is used as origin. The chiral vector $\mathrm{C}$ is pointed from the

53 first atom toward the second one and is defined by the relation: $\mathrm{C}=\mathrm{n} \alpha 1+\mathrm{m} \alpha 2$ where $\mathrm{n}$ and $\mathrm{m}$ are

54 integers. The values, $\alpha 1$ and $\alpha 2$, are the unit cell vectors of the two-dimensional lattice formed by the

55 graphene sheets. The direction of the nanotube axis is perpendicular to this chiral vector as described

56 previously [9-10]. The very long CNTs are generally metallic. CNTs are very hard, strong and chemically

57 stable materials; very fast thermal and electrical conductors. The two types of CNTs are components of

58 different devices which are avidly researched in industry today. Semi-conducting carbon nanotubes are

59 applied in memory devices and sensors whereas metallic carbon nanotubes are applied in electrode

60 materials of cells and electromagnetic shields. Thus, there is a need for separating the types and

61 isolating those of specific chirality. There are various methods of producing the CNTs such as chemical

62 vapor deposition and arc discharge on catalysts. Some methods do not produce a high amount of the

63 semiconductor types and there are some methods of seeding the desired types, but the yields are

64 limited [2-3].

65 The semi-conducting species of carbon nanotubes have been separated by density gradient ultra-

66 centrifugation $[5,11]$ ion exchange, gel chromatography and electrophoresis [12-15]. Separation

67 methods also include chiral nano-tweezers [16]. The yields are small from ion exchange and results

68 from differential gradient ultracentrifugation yield small fractions, although chiral enantiomers are

69 separated. The nanotubes are solubilized in water by binding with anionic surfactants such as sodium

70 dodecyl sulfate SDS [12] and DNA [13], and processes separate on the basis of surface charge which 
71 varies with the sizes of the particles. Since CNTs can bind with dextran [15], column and ATPE (aq. 2

72 phase extraction) methods using dextran [17] or related materials are used. Agarose or Sephacryl and

73 related supports achieve separation of semiconducting CNTs by size [17-19] and other ATPE methods

74 are described [20-22]. Novel solvent systems and new agents for selectivity can be readily used in CCC.

75 Methods to achieve mono disperse species with increased mass recoveries are needed. CCC can be

76 investigated to achieve this.

77 The spiral-design CCC rotors, the spiral tubing support and mixer-settler spiral disk rotors have proved

78 useful for the separation of proteins, the large bio-molecules [24-26]. The primary advantage of using

79 these rotors in HS-CCC is that the stationary phase retention of the high viscosity ATPS is high, $60-80 \%$ at

80 moderate flow rates which is much better than the other types of CCC rotors. This has made possible

81 the application to large molecules [27]. CCC does not use expensive solid column packing that takes up

82 volume and adsorbs mass. The higher volume of the stationary phase simply holds more sample mass.

83 The system is versatile in that either phase can be utilized as the mobile phase to maximize the

84 separation.

85 The chromatography samples were applied to the field-effect transistor (FET) circuits according to the 86 procedures of Fuzbien Technology Institute [28-29] to determine semiconductor activity.

87

\section{$88 \quad 2.0 \quad$ Material and Methods}

892.1 Materials. Dextran MW 75,000 was obtained from Fisher Scientific (Boston, MA) (Tokyo Chemical

90 Co. manufacturer) or Spectrum Chemical (Gardena, CA), polyethylene glycol MW 8000 was from Fisher

91 Scientific or Spectrum Chemical, sodium cholate, sodium deoxycholate and sodium dodecylsulfate were

92 from Fisher Scientific or Spectrum Chemical, carbon nanotube dispersion of an enriched nanotube

93 preparation (designated $6,5 \mathrm{i}$ by the manufacturer) in $0.2 \%$ sodium deoxycholate was obtained from 
South West Nanotechnologies Inc. (now Chasm Advanced Materials, Norman, OK). A sample of 6,5i

95 powder from the same manufacturer was also used. Since the concentrations of individual components of the $(6,5 i)$ preparation have not been quantified by the manufacturer and there are no methods to

97 quantify distinct SWCNT chiral species, we have relied on spectral scans to identify these species. A dispersion of 3\% CNTs in 3\% PVP (polydivinylpyrrolidone) was from Cheap Tubes, Inc. Grafton, VT. Also from the same manufacturer a powder of short SWNTs >90wt\% was used. From Nano-C, Westwood, MA, a powder of as-produced SWCNT APT25, < 1 micron in length was provided. Water was purified in 101 a Neu-Ion system (Baltimore, MD) or HPLC water was obtained from Fisher Scientific. one mixer-settler rotor (17.5 cm OD, cat. no. 205-10001, CC Biotech, Rockville MD)[30]. The early experiments were done with the previously reported rotor, made of stacked poly-carbonate disks [10]. Each disk has 4 interleaved spirals that the flow goes through serially, then to the next disk. The flow channel groove in the disk has mixing and settling sections with a glass bead in every $4^{\text {th }}$ section. The total volume in the rotor with 6 layers is $84 \mathrm{ml}$. We performed experiments with the rotor made with 7 or 5 layers at times. There were difficulties with leaking and the channel underneath each disk was made too shallow such that another gasket was designed and inserted to space out the sealing gasket to 110 allow the liquid flow. However, there were problems with high back pressure and air getting in the 111 rotor.

112 To correct these problems we designed and made a new rotor as shown in Fig. 1. It has thicker top and 113 bottom metal plates to apply stronger even pressure on the plastic disks. The mixer-settler plastic disk 114 had the return-channels underneath deepened so an extra sheet or gasket was eliminated. The 115 segment divisions or pins in the flow channels were straightened out as it was discovered these were 116 not centered throughout the channel. The new disk is shown in Fig. 2. The volume of 6 layers was 117 around $60 \mathrm{ml}$. The lower volume could be due to the 'pins' or protrusions in the channels which are 
slightly larger in these printed disks. The holes in the disks and gaskets were made slightly wider, as

119 well. As a result the performance was a lot better, with lower pack pressure and no difficulty with air bubbles forming. The rotor weighs $2.2 \mathrm{Kg}$ which is $0.6 \mathrm{Kg}$ more than the first rotor.

121 The inlet and outlet PTFE flow tubing, 1/32-in ID (Zeus Industrial Products, Orangeburg, SC) are

122 connected to the top flange (metal plate) and bottom plate, respectively with metal nuts (compression

123 screws, each with a stainless steel flange and plastic ring, IDEX Health and Science, Chicago, IL) and

124 passed into the rotor shaft and out the bottom into the central axis and out the top of the chamber

125 where they are clamped to prevent twisting. The other side of the rotor arm has counterbalancing

126 metal rings equal in weight to the mixer-settler rotor, placed the same distance from the center axis as

127 described previously [30].

128 The solvent is pumped from a gradient dual pump system (DGS-200 automated delivery system, D-Star

129 Instruments, Manassas, VA, controlled with Clarity software). The flow passes through a manifold with a

$13010-\mathrm{ml}$ sample loop valve and another valve with Helium for changing the flow to gas pressure to clear

131 out the rotor contents. The solvent flow is then connected to the in-flow tubing to the CCC instrument.

132 The out-flow from the instrument central axis goes to a fraction collector (Pharmacia or Bio-Rad).

$133 \quad 2.3$ Solvent system. The aqueous two-phase solvent system consisted of polyethylene glycol MW 8000

134 (PEG) and dextran MW 75,000 (DEX) containing sodium deoxycholate (SDC). The gradient of sodium

135 dodecylsulfate (SDS) from 0 to $0.7 \%$ in the upper PEG rich phase as the mobile phase served to elute the

136 carbon nanotubes in the chromatography. The volumes of the stock solutions listed in Table 1 were

137 mixed in a separatory funnel and the phases separated after some time, $45 \mathrm{~min}$. The resulting phases

138 distributed to $351 \mathrm{ml}$ upper phase (UP) and $129 \mathrm{ml}$ lower phase (LP) for SS\#1. The solvent composition,

139 except for a numeric error in the solvent system preparation that was corrected, and the gradient

140 method as reported previously were followed [8, 21]. 
$141 \quad 2.4$ Sample preparation. The best method developed for sample preparation of powdered CNTs was

142 to sonicate (Branson 450 UltraSonic) with a probe, short pulses in 2 min segments approximately 3

143 times, a $1 \%$ solution of CNT powder followed by $5 \mathrm{~min}$ in a water bath sonicator. A suspension of $10 \mathrm{mg}$

144 CNTs in $0.5 \mathrm{ml} 5 \%$ sodium cholate (SC) and $0.5 \mathrm{ml} 5 \% \mathrm{SDC}$ is thus sonicated and $0.25 \mathrm{ml}$ is removed and

145 added to $3 \mathrm{ml} \mathrm{UP} \mathrm{SS \# 1} \mathrm{and} 3 \mathrm{ml}$ LP SS\# 1, vortexed and allowed to settle into 2 phases, The upper

146 phase is removed and $1 \mathrm{mI}$ UP SS\#1 is added and the total is loaded into the CCC. This was the

147 procedure for the $2.5 \mathrm{mg}$ sample load.

148 In the early experiments, dispersions provided by others were extracted according to the previously

149 published method using the solutions described in Table 3. A $0.5 \mathrm{ml}$ or $1 \mathrm{ml}$ amount of the CNT

150 dispersion (approximately $0.1 \%$ CNTs $6,5 \mathrm{i}$, in $0.2 \% \mathrm{SDC}$ ) is extracted and loaded via the sample loop.

151 Generally, the sample prep was done by combining $0.5 \mathrm{ml}$ carbon nanotube dispersion (CNTs) $+0.5 \mathrm{ml}$

$15210 \% \mathrm{SC}+4 \mathrm{ml}$ UP SS\#3 $+0.5 \mathrm{ml}$ LP SS\#3, vortex, centrifuge $1000 \mathrm{rpm}, 3 \mathrm{~min}$. The upper phase is

153 removed and to this is added $1 \mathrm{mI} \mathrm{UP} \mathrm{SS \# 3} \mathrm{and} \mathrm{all} \mathrm{is} \mathrm{loaded} \mathrm{into} \mathrm{the} \mathrm{system.}$

1542.5 Elution mode and other operating conditions. The rotor is filled with lower phase at a rate of 0.5

$155 \mathrm{ml} / \mathrm{min}$. Sample is loaded in a 10-ml loop; centrifugation at $990-1000 \mathrm{rpm}$ is started and then sample in

156 the loop is injected online. Next the upper phase \#1 is pumped with flow of $0.5 \mathrm{ml} / \mathrm{min}$. The gradient is

157 started when the dark colored sample has fully entered the rotor. A gradient of UP SS\#1 A to UP SS\#2 B

158 is made over an 8-hr period for a 5 layer rotor or more for increased layers in the rotor (Table 2).

159 Fractions of $8 \mathrm{~min}$ are collected. Elution mode $=\mathrm{U} \circ \mathrm{H},(\mathrm{CCW}$ centrifugation) which means sample and

160 mobile phase flow enter through the bottom of rotor in head to tail direction [30].

1612.6 Analysis of Fractions. Generally a $0.5 \mathrm{ml}$ aliquot is taken out of the vortexed fraction to include

162 both phases and $0.5 \mathrm{ml} 2 \%$ aq. SDS is added to dissolve all phases and make a clear solution.

163 Absorbance at 2 wavelengths are read with water as the blank in a Cary 3E VC-Vis Spectrophotometer 
164 (Varian, division of Agilent, Santa Clara, CA). The absorbance is plotted of the fractions. Fractions

165 containing black solids (soot) or dark solutions and colors are noted. When concentrated fractions were 166 achieved in the CCC runs, spectra of these were measured from 300 to $900 \mathrm{~nm}$ to analyze the peaks 167 indicating the chiral species.

1682.7 Semiconductor activity. The SWCNTs are applied to circuits printed on standard 4" silica

169 semiconductor wafers (92 circuit size) [28-29]. For measuring field effect transistor impedance, the CNT 170 coated circuits are treated with 2 ul Di water for 20-30 sec to obtain baseline values for each circuit.

171 After baseline accrual, $2 \mathrm{ul}$ polybrene $(50 \mathrm{ul} / \mathrm{ml}$ in water $)$ is added and an increase in impedance is

172 measured to saturation. The levels of saturation are compared to a standard curve for the polybrene.

173 Normalized impedance as a function of voltage applied is determined as a measurement of

174 semiconductor activity of the SWCNTs in the CCC fraction.

\section{$175 \quad 3.0$ Results}

$176 \quad 3.1$ Early experiments.

177 For a good characterization of the spiral CCC process, it is best to perform separations of the purest

178 carbon nanotubes available, so we started on a liquid dispersion 6,5i from SouthWest Nanotechnologies 179 that was composed of mostly 6,5 chiral species with a range of lengths of the nanotubes. The $0.1 \%$ CNTs 180 in $0.2 \%$ SDC solution/dispersion was dark, mostly black, with some passage of light. Extracts of $2 \times 0.5$ 181 or $1 \mathrm{ml}$ dispersion were made and run in CCC. The pre-load extraction procedure was modified from 182 using SS\#3 to SS\#4 in various experiments. The result of a sample without the extraction is shown in Fig.

183 3. This run had more mass of sample and included right after the solvent front, an absorbing peak that 184 had not been extracted away.

185 Two $\mathrm{ml} \mathrm{CNT}$ solution and $1 \mathrm{ml} \mathrm{10 \% SC}$ and $2 \mathrm{ml} \mathrm{LP \# 1}$ were sonicated and the total volume was loaded. 186 The convex gradient (Table 2) was run. In Fig. 3 the dotted line indicates \% B or UP of SS\#2. After the 
solvent front the fractions were grey and a strong UV absorbing peak was measured. Nevertheless,

188 green fractions are noted at fraction 47 and purple starting at 50 . Subsequent runs with different

extractions and more volume looked like this run with green fractions followed by purple fractions. The colored fractions had CNT-FET reactivity (data not shown). After the chromatography the fractions settled into distinct phases with color in the lower phase, which contain the separated carbon at the solvent front and more dark particles in the stationary phase left in the rotor, eluted after the

194 pump out. After chromatography runs it is necessary to wash the rotor with many column volumes of 195 water to remove SDS that may remain. The SDS is harder to remove from the new rotor made with a 196 different plastic than the original polycarbonate. The bright green fractions followed by purple fractions 197 indicated that the 6,5 chiral species was the major type and the chiral type of green color was removed. 198 We did not have concentrated enough solutions to perform spectral scans.

\subsection{Separation of SouthWest Nanotechnologies Powders.}

200

201

During the sample preparation, we observed that the spectrum of the solubilized powder corresponded with a 6,5i product spectrum (on the website of Chasm Advanced Materials) and shows the presence of chiral species of CNTs. Figure 4 shows that the spectrum has the same peaks with the exception that the peak corresponding to 6,4 is higher. The complex peak labeled as 7,5 and 7,6 can also include others such as 8,3 [8]. A 5 mg amount of powder from SouthWest Nanotechnologies, SWeNT ${ }^{\circledR}$ SG6,5i-L43 was suspended at $10 \mathrm{mg} / \mathrm{ml}$ and sonicated as described above. A volume of $250 \mu \mathrm{l}$ of the CNTs was taken and added to SS\#1, $3 \mathrm{ml}$ of each phase, as described. After equilibration the upper phase was removed and another amount of $1 \mathrm{ml}$ upper phase was added and the total loaded. A linear gradient from 0 to $100 \% \mathrm{~B}$ was run at $0.5 \mathrm{ml} / \mathrm{min}$ over $540 \mathrm{~min}$ in the new mixer-settler rotor with 6 layers. The absorbance of the fractions was read and plotted in Fig. 5. All of the tubes were photographed and are shown in the Supplementary Material. The fractions after the solvent front had gray upper phase and 
211 black fluffy precipitate at the interface and comprised the highest UV peak in Fig. 5. This was followed

212 by clear fractions, two pink fractions came out, 48-49, followed by clear fractions then light blue

213 fractions, a fraction that was dark yellow or green then purple fractions. Figure 6 shows photos of the

214 colored fractions which have settled into 2 phases with the lower dextran phase containing the colored

215 chiral CNTs. The carbon nanotubes with surfactants bind to dextran in the stationary phase so all elute

216 as micelles [28]. Upon standing these fractions separate into two phases with the CNTs in the lower

217 phase. This is why the fractions containing the analytes have significant stationary phase as seen in the

218 pictured test tubes. Usually the fractions have only the mobile phase (upper phase).

219 These data suggest that the separation of the CNTs into enriched chiralities was successful. To further 220 examine the CNTs in each fraction, we performed spectral analysis. Shown in figure 7 is the overlayed

221 spectra of those fractions. From the spectral analyses, the purple fractions are the major chiral species 222 of 6,5 and fraction 58 could be a mixture of 7,4 metallic with the 6,5 semiconducting CNTs. The peak 223 maxima corresponding to the 6,4 species was not present in these fractions thus, was separated away.

224 We assessed the impedance of the above fractions and those are shown in Table 4. The highest level of 225 impedance is not with the mass of CNTs at the solvent front, but at the colored fractions. In making a 226 rough specific activity determination, the highest activity corresponds to the blue fractions $54-57$. The 227 major 6,5 chiral species $(570 \mathrm{~nm})$ are not as strongly semiconducting as the other fractions.

228 Interestingly, the peak activities are in fractions 56 and 57, which are blue. Figure 7 shows these 2

229 fractions and there are 2 major peaks at 670 and $835 \mathrm{~nm}$, respectively. The $670 \mathrm{~nm}$ absorbance peak 230 could correspond to 7,5 or 7,6 or other chiral species identifiedto have similar maxima. Fraction 58 231 could be mixed with 6,5 and metallic CNTs, resulting in less activity.

\subsection{Separations of CNTs provided by Cheap Tubes, Inc. and Nano-C}


234 dispersions and powders were analyzed. The results of using the new rotor with the same linear

235 gradient are shown (Fig. 8). Such clear separated colors were not noted which indicates that besides the 236 composition being different, the samples were not pre-processed to concentrate any chiral species. In

237 the case of samples from Cheap Tubes, these are processed to provide short length of single wall-double 238 wall CNTs 90\% wgt. From Cheap Tubes we first obtained a dispersion of 3\% CNTs in 3\% PVP

239 (polydivinylpyrrolidone) which was very thick. We made the sample preparation with a small sample 240 size of $100 \mathrm{ul}$, added to SS\#4. Much black color was in the upper phase which was removed but still 241 black color was at the solvent front. An intriguing broad retained peak of fractions 46-54 with 2 maxima 242 at fractions 48 and 50 may represent some separation. The early part of the peak had a yellow color and 243 may be a chiral species.

244 In Fig. 9 is a CCC run in the previous rotor of a dispersion made of a powder (As-produced SWCNT 245 APT25, < 1 micron in length, Nano-C). A 1\% solution in $10 \%$ SDC was sonicated and combined with equal 246 volume of $10 \%$ SC. A small volume, $300 \mathrm{ul}$ was added to $1.5 \mathrm{ml}$ UP and $1.5 \mathrm{ml}$ LP SS\#1, sonicated in a bath 247 and loaded. The major peak of CNTs with yellow/green at beginning and the rest dark green elutes 248 earlier than the other samples. There is less absorbance in the $40-50 \%$ part of the gradient where CNTs 249 come out in the SouthWest Nano samples.

\section{$250 \quad 4.0$ Discussion}

251 In summary, we are excited about these results and believe that with applying this linear gradient with 252 an appropriate mass load there is a worthwhile comparative analysis of the preparations from the 253 various sources. The gradient can be modified to maximize separations between the carbon nanotube 254 chiral species. Also, with the availability of an instrument that provides low temperature which is 255 known to optimize the partitioning of chiral species, the separations may be further optimized. The CNT 
powders and dispersions supplied from various manufacturers gave different results. The SouthWest

257 Nanotechnologies product was already pre-fractionated to an enriched content of a particular chiral

258 species that was significantly purified here. The other samples require processing step(s) to increase the

259 concentration of major chiral species that could be isolated in high purity in this chromatographic step.

260 The spiral CCC instrument is a convenient method to purify the many species of carbon nanotubes for

261 their investigation in research laboratories. The recent redox sorting extraction [32] and ionic liquids

262 [33] can be adapted in this versatile technology. The CCC method can serve as an analytical technique

263 as well as a preparative method.

264 A very interesting result is that the major species purified, the 6,5 chiral species did not have the most

265 potent semiconducting activity in field-effect transistors. Instead a light blue chiral type seems to be

266 more potent. Further analyses by UV near IR spectroscopy will identify which chiral type. It will be

267 interesting to study as many chiral species as possible in the future to apply the ones most suitable for a

268 device.

269 The new mixer-settler rotor made in this project is performing better than the first rotor. Thus this

270 improved development in CCC will be beneficial to introduce in materials science research. To this end

271 fabrication of a scaled-up instrument with cooling in the rotor chamber is underway at present.

\section{Acknowledgement}

273 This work was supported by National Science Foundation grant 1548957 and the authors would like to

274 thank Dr. Ming Zheng of the Materials Science and Engineering Division of the National Institute of

275 Standards and Technology for helpful discussions. Current affiliation of S. Stefansson is HeMemics

276 Biotechnologies Inc, Rockville MD 20850.

277 The mixer-settler spiral disk rotor is manufactured by CC Biotech LLC under a license L 144-2007 from

278 the National Institutes of Health. 
280 1. Davenport, M. 'Much Ado About Small Things', Chem. \& Eng. News CEN ACS.ORG Jun 8, 2015, 10-15.

281 2. Yang, F., Wang, X., Zhang, D., Yang,J., Luo, D., Xu, Z., Wei, J, Wang, J-Q., Xu, Z., Peng, F., Li, X., Li, R., 282 Li, Y., Li, M., Bai, X., Ding, F.,Chirality-specific growth of single-walled carbon nanotubes on solid alloy catalysts, Nature (2014) 510: 522-524, doi: 10.1038/nature13434.

3. An, $H$, Kumamoto, $A$, Takezaki, $H$, Ohyama, $S$, Qian, $Y$, Inoue, $T$, Ikuhara, $Y$, Chiashi, S, Xiang, $\mathrm{R}$, and Maruyama S, Chirality specific and spatially uniform synthesis of single-walled carbon nanotubes from a sputtered Co-W bimetallic catalyst, Nanoscale, (2016) 8:14523-14529 doi: 10.1039/C6NR02749K.

4. Che,Y., Chen, H., Hui, G., Liu, J., Zhou, C. Review of Carbon Nanotube Nanoelectronics and Macroelectronics, Semicond. Sci. Technol. (2014) 29:073001, doi:10,1088/0268-1242/29/7/073001.

5. Hersam, M.C., Progress towards monodisperse single-walled carbon nanotubes, Nature, Nanotech. (2008) 3: 387-394.

6. Knight ,M., Finn, T. M., Zehmer, J., Clayton, A., Pilon, A. Spiral counter-current chromatography of small molecules, peptides and proteins using the spiral tubing support rotor. J. Chromatog.A (2011)1218: 6148-6155, doi:10.1016/j.chroma.2011.06.007

7. Cai, Y., Yan, Z.H. Zi, M, Yuan, L.M. Preparative Purification of Single-Walled Carbon Nanotubes by High Speed Countercurrent Chromatography, J. Liq. Chromatog. \& Rel. Technol. (2009) 32: 399-406. Single Wall Carbon Nanotubes by Countercurrent Chromatography, Anal. Chem. (2014) 86: 3980- 
9. Belin, T. and Epron, F.,. Characterization methods of carbon nanotubes: a review. Materials Science and Engineering: B, (2005)19: 105-118

10. Komatsu N, Wang F. A comprehensive review on separation methods and techniques for singlewalled carbon nanotubes. Materials. (2010) Jun 30;3(7):3818-44

11. Ghosh, S, Bachilo, S.M., Weisman, R.B. Advanced sorting of single-walled carbon nanotubes by 305 nonlinear density-gradient ultracentrifugation, Nature Nanotech. May 9, (2010) doi:

12. Bonard, J.M., Stora, T. Salvetat, J.P., Maier, F. Stockli, T. Duschl, C., Forro, L, deHeer, W.A, 10.1038/NNANO.2010.68. Chatelain,D. Purification and Size-Selection of Carbon Nanotubes, Adv. Mater.(1997) 9:827-831.

13. Zheng, M. Jagota,A., Strano,M.S., Santos,A.P., Barone, P., Chou, G., Diner,B.A., Dresselhaus, M.S.

14. Doorn, S.K, Strano, M.S. O'Connell, M.J., Haroz, E.H., Rialou, K.L., Hauge, R.H., Smalley, R.E., Capillary Mclean, R.S., Onoa, G.B, Samsonidze, G.G., Semke, E.D., Usrey, M., Walls D.J. Structure-based carbon nanotube sorting by sequence dependent DNA assembly. Science 302, 1545-1548 (2003).

15. Lukaszczuk, P. Rummeli, M.H., Knupfer, M. Kalenczuk, R.J., Borowiak-Palen, E., Surfactant free fractions of metallic and semiconducting single-walled carbon nanotubes via optimised gel chromatography, Materials Research Bulletin (2012) 47:687-691

16. Peng, X, Wang F., Bauri, A.K., Rahman, A.F.M.M., Kamatsu, N. Optical Resolution of Single-Walled Carbon Nanotubes through Molecular Recognition with Chiral Diporphyrin Nanotweezers, Chem. Lett (2010)39: 1022-1027, doi:10.1246/cl2010.1022.

17. Stobinski, L, Polacek, D, Rebilas, K, Mazurkiewicz, J, Wrzalik, R, Lin, H-M, Tomasik, P. Dextran Complexes with SWCNT, Polimery/Polymers (2008) 53:571-575. 
18. Tanaka, T, Urabe Y., Nishide, D., Kataura, H., Continuous Separation of Metallic and Semiconducting Carbon Nanotubes Using Agarose Gel, Applied Physics Express, (2009)2:125002

19. Flavel, B.S., Moore, K.E., Pfohl, M, Kappes, M.M., Heinrich, F., Separation of single-walled carbon Nanotubes with a gel permeation chromatography system (2014) ACS Nano, 8:1817-1826.

20. Moore, K.E., Pfohl, M., Kuebel, C.K., Tune, D.D., Heinrich,F., Dehm,S., Chakradhanulam V.S.K., Kubel, C.,Krupke, R., Flavel, B.S., Sorting of Double-Wall Carbon Nanotubes According to their Outer Wall

21. Khripin, C.Y., Fagan, J.A. Zheng, M., Spontaneous Partition of Carbon Nanotubes in PolymerModified Aqueous Phases, J. Am. Chem. Soc. (2013) 135: 6822-6825, dx.doi.org/10.1021/ja402762e

22. Tang, M.S.Y., Show, .PL., Lin, Y.K., Woon, K.L., Tan, C.P., Ling, T.C., Separation of single-walled Carbon Nanotubes using Aqueous Two-phase System, (2014) Sep. and Purif. Technol. 125:136-141.

23. Subbaiyan, NK, Cambre, S, Parra-Basquez, ANG, Haroz, EH, Doorn, SK, Duque, JG, Role of Surfactants 335 and Salt in Aqueous Two-Phase Separation of Carbon Nanotubes toward Simple Chirality Isolation,

24. Ito, Y., Clary, R. Powell, J, Knight, M, Finn, T, Spiral Tube Support for high-speed countercurrent chromatography. J. Chromatog. \& Rel. Technol, (2008) 31: 1346-1357.

25. Ito, Y., Clary, R., Sharpnack, F., Metger, H. and Powell, J.; Mixer-settler counter-current chromatography with multiple spiral disk assembly, J Chromatography A, (2007) 1172: 151-159.

26. Ito. Y., Qi, L., Powell, J., Sharpnack, F., Metger, H., Yost, J. ; Mixer-settler countercurrent chromatography with a barricaded spiral disk assembly with glass beads, J. Chromatography A, (2007) 1151:108-114. 
27. Ito, Y., Knight, M., Finn, T.M., Spiral Countercurrent Chromatography, Review, J Chrom. Sci.(2013) 51: 726-738, doi:10.1093/chrom sci/bmt058.

28. Stephansson, S., Knight,M., Ahn, S. N.,'Specific Binding of Alzheimer's A $\beta$ Peptide Fibrils to SingleWalled Carbon Nanotubes, Nanomat. Nanotech. (2012) Vol. 2 Art. 11:2012.

29. Stefansson S, Stefansson LA, Chung SW, Ko K, Kwon HH, Ahn SN. 'Evaluation of aromatic boronic 349 acids as ligands for measuring diabetes markers on carbon nanotube field-effect transistors, $J$. Nanotech. (2012) Article ID 371487.

30. Knight, M., Finn, T.M , Spiral countercurrent chromatography studies using the spiral disk assembly, J. Liq. Chromatog. \& Rel. Technol. (2009) 32: 2669-2685.

31. Shen, C.W., Yu, T. (2007) Protein separation and enrichment by countercurrent chromatography 354 using reverse micelle solvent systems, J. Chromatogr. A, 1151 164-168.

355 32. Gui, H, Streit, JK, Fagan, JA, Hight Walker, AR, Zhou, C, Zheng, M, Redox Sorting of Carbon 356 Nanotubes, (2015) NANO Lett., doi:10.1021/nl504189p

357 33. Smirnova, SV, Samarina, TO, Pletnev, IV, Hydrophobc-hydrophilic ionic liquids for the extraction and 358 determination of metal ions with water-soluble reagents, Anal. Methods, (2015) 7:9629-9635, doi: 10.1039/C5AY01914A. 
362 Figure 1 Mixer-settler spiral disk CCC rotor, ver.2. The assembled separation rotor, left; view without 363 the gear. Next to shaft is seen an opening for the compression screw where the liquid flow enters. Six 364 disks are sandwiched between black Viton gaskets. Right; bottom view is seen where circumference 365 screws are held with double nuts to provide more even pressure.

366 Figure 2 New mixer-settler spiral disk fabricated by high density stereo-lithography with epoxy-like 367 resin (SOMOS NeXT) with wet-dry blast surface treatment. Left is top surface showing the 4 368 interweaved flow channels with the retaining 'pins' defining segments where glass beads (not shown) 369 are placed in every 4th segment (mixing section). Right is the underside showing the channel from each 370 spiral going to the next spiral or to the hole for flow to disk beneath. A Viton gasket (not shown) with 371 the one hole over the opening to lower disk is placed between the disks. The photos are not the same 372 scale.

373 Figure 3 Counter-current separation of a dispersion of single-walled carbon nanotubes using the 374 polymer phase solvent system with the SDS surfactant convex gradient (upper phase of SS\#2, shown as $375 \%$ B). Further details are in the 'early experiments' section 3.1. The previous mixer-settler CCC rotor 376 was used [8].

377 Figure 4 Ultraviolet wavelength reading from 300 to $900 \mathrm{~nm}$ of the SWCNT solution $(6,5 i)$ prepared as

378 described in the text, section 3.2. The peaks are labeled with the chiral species (in parenthesis) that are 379 known to have that maxima according to the company's website (www.chasmadvancedmaterials.com).

Figure 5 Plot of the absorbance measurements of the fractions of the separation of the So West Nano

382 CNTs prepared from a powder as described in section 3.2. The sample load of $2.5 \mathrm{mg}$ was separated in 383 the new mixer-settler rotor using a linear gradient of \% B in the mobile phase. . 
385 Figure 6 Close up photo of the colored fractions. The colors were bright and concentrated enough to 386 provide spectra that is presented in Figure 7. This was the most clear looking results that were found, 387 due to not overloading the system and showed the presence of various colored CNT species.

389 Figure 7 Overlay of spectra taken of the colored fractions. The major peak of purple color $(570 \mathrm{~nm})$ 390 corresponding to 6,5 has a smooth symmetrical peak, missing the hump in the unpurified sample (Fig.

391 4). The blue fractions, 54 through 58 have peaks corresponding to other chiral species, such as 8,4

392 (small amount around $605 \mathrm{~nm}$ ) and 7,5 or 7,6 $(670 \mathrm{~nm}$ ) that are removed from the 6,5 chiral species.

394 Figure 8 Plot of the spCCC separation of the dispersion of CNTs in PVP, 100ul or approximately $0.3 \mathrm{mg}$ 395 in the new mixer-settler rotor using a linear gradient. Results are described in section 3.3.

397 Figure 9 Counter-current separation of a CNT dispersion made from a Nano-C powder in the first mixer-

398 settler rotor as described in section 3.3. The major peak emerging after fraction 26 was mostly green 399 indicating a different composition of CNTs. 


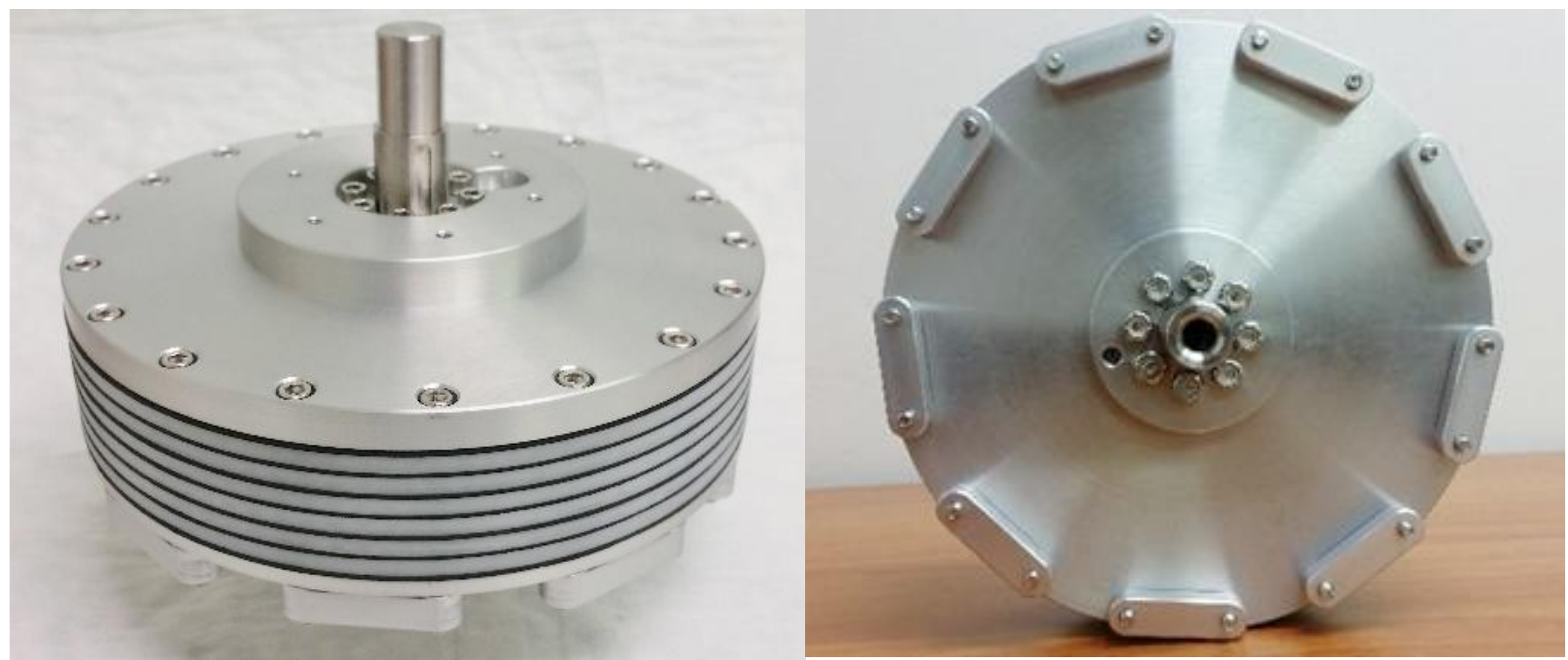

Figure 1 


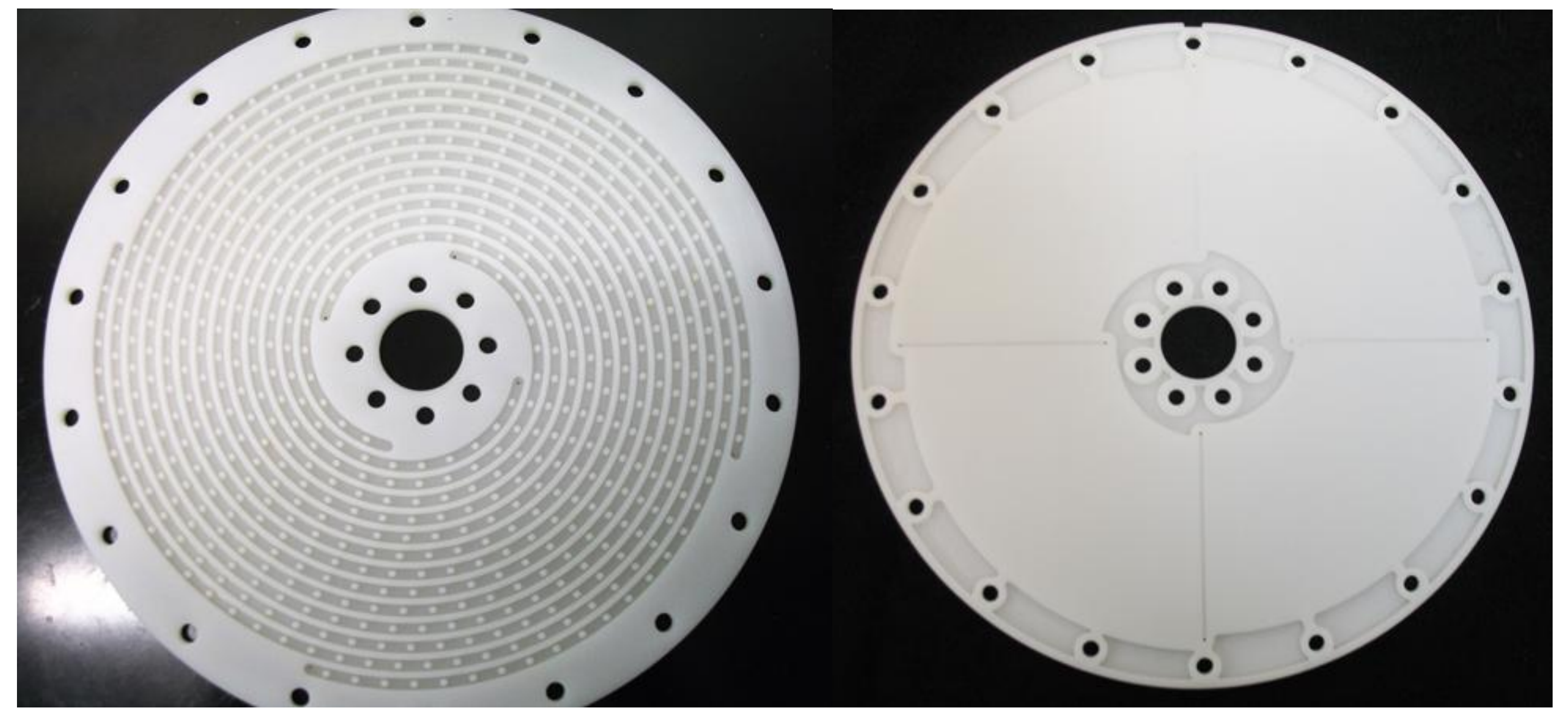

Figure 2 


\section{spCCC Carbon Nanotubes [SoWest Nano] $(2 / 3 / 16)$}

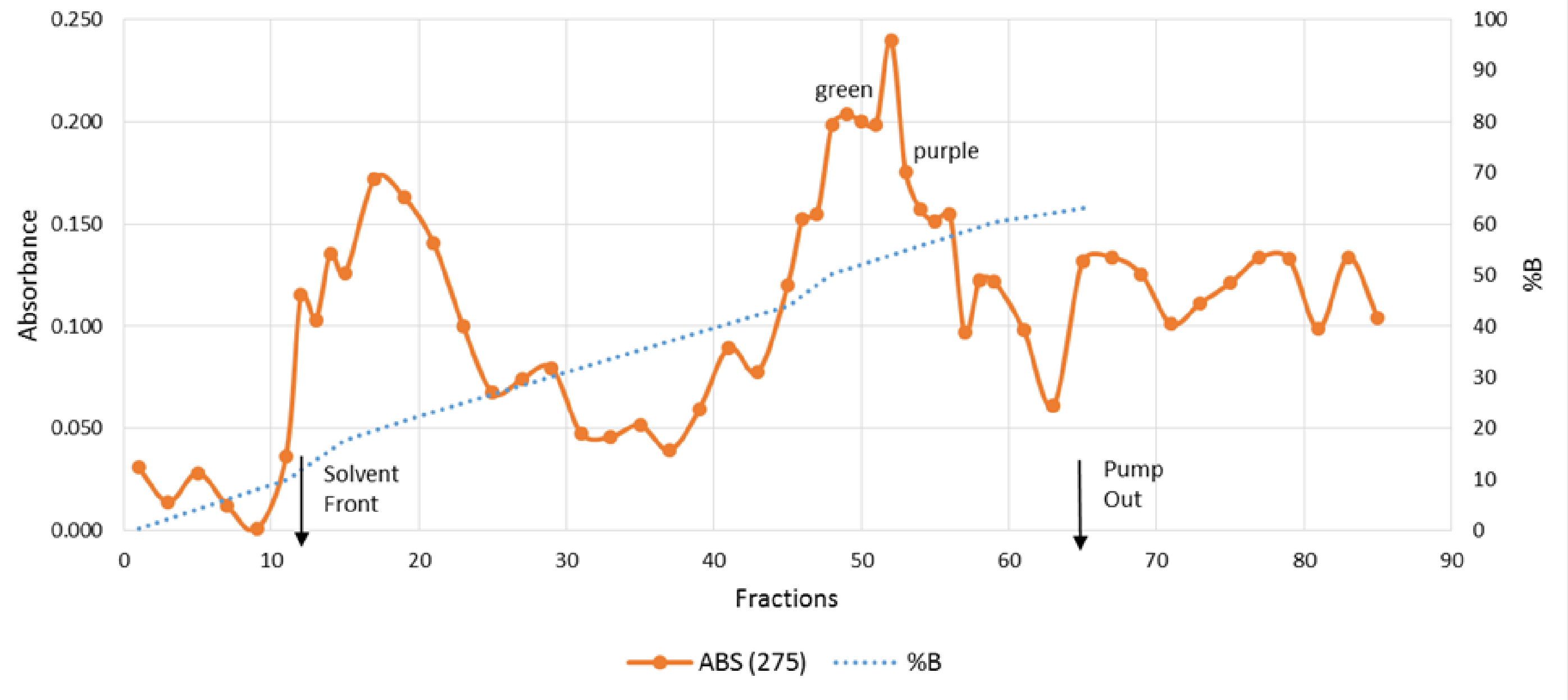

\section{Figure 3}




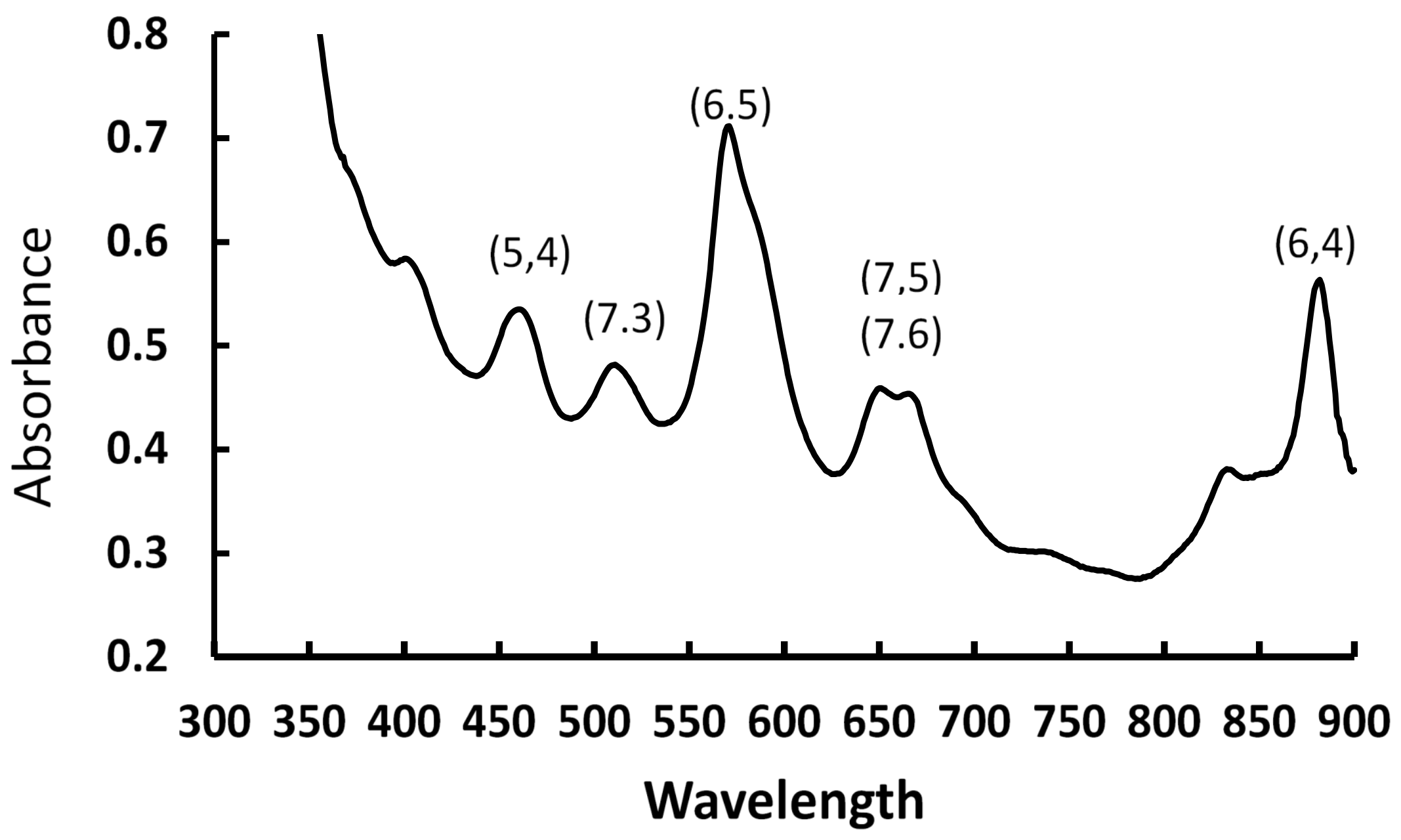

Figure 4 
spCCC CNT Separation SWNano (2.5mg) 06-29-16

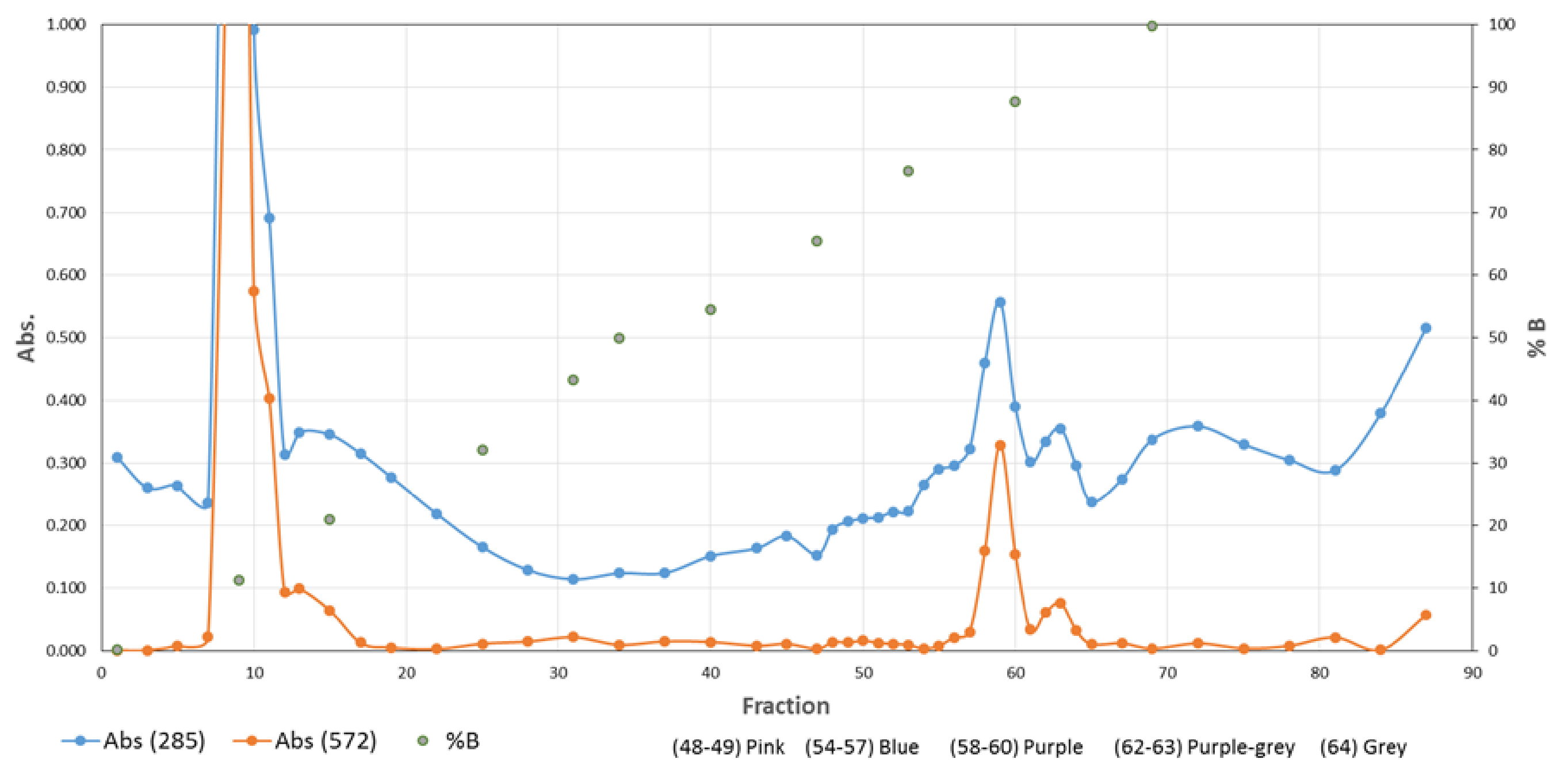

\section{Figure 5}




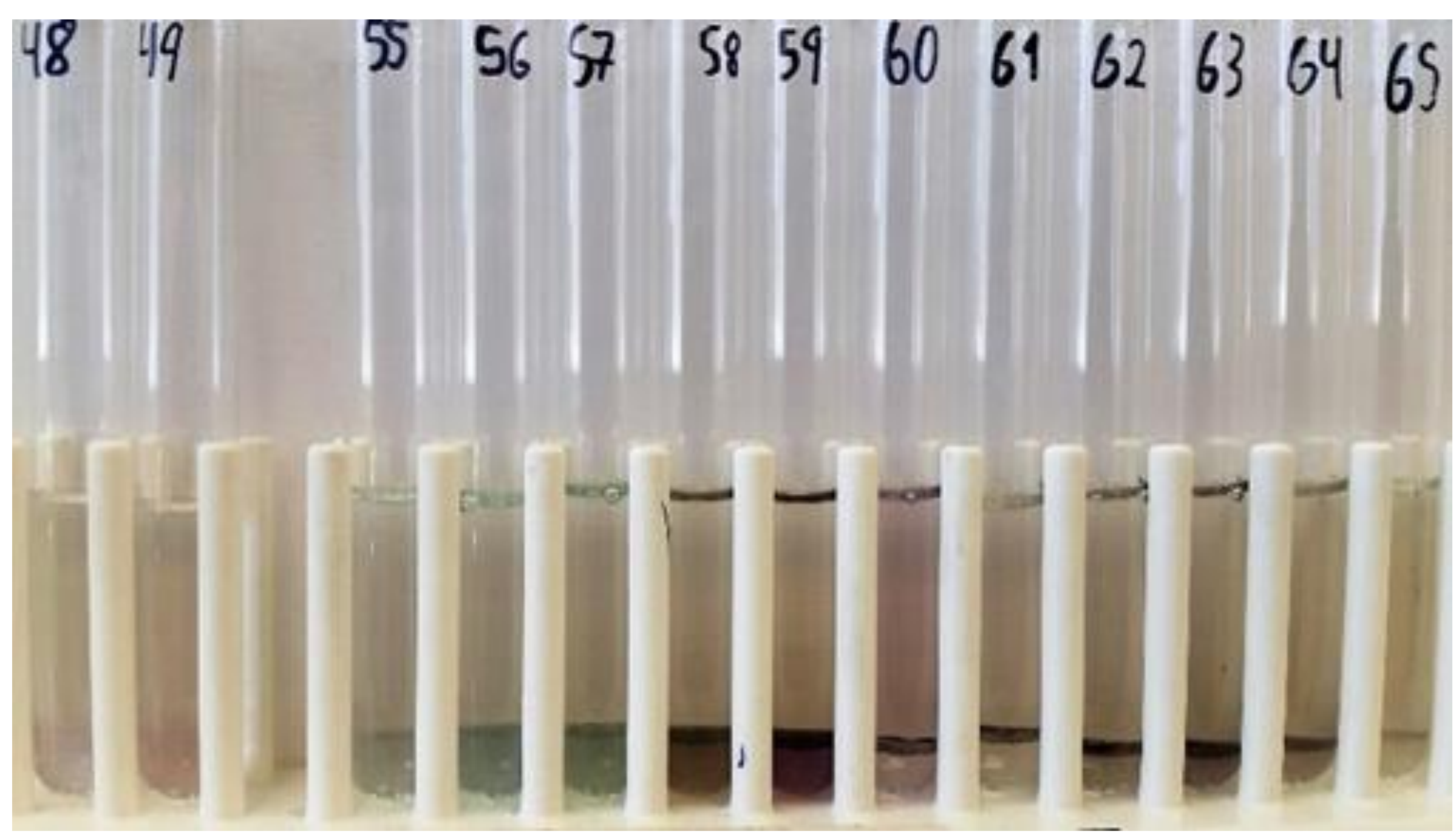

Figure 6 


\section{SPECTRA FOR FRACTIONS 54 to 60 (spCCC 06/29/16)}

Figure 7

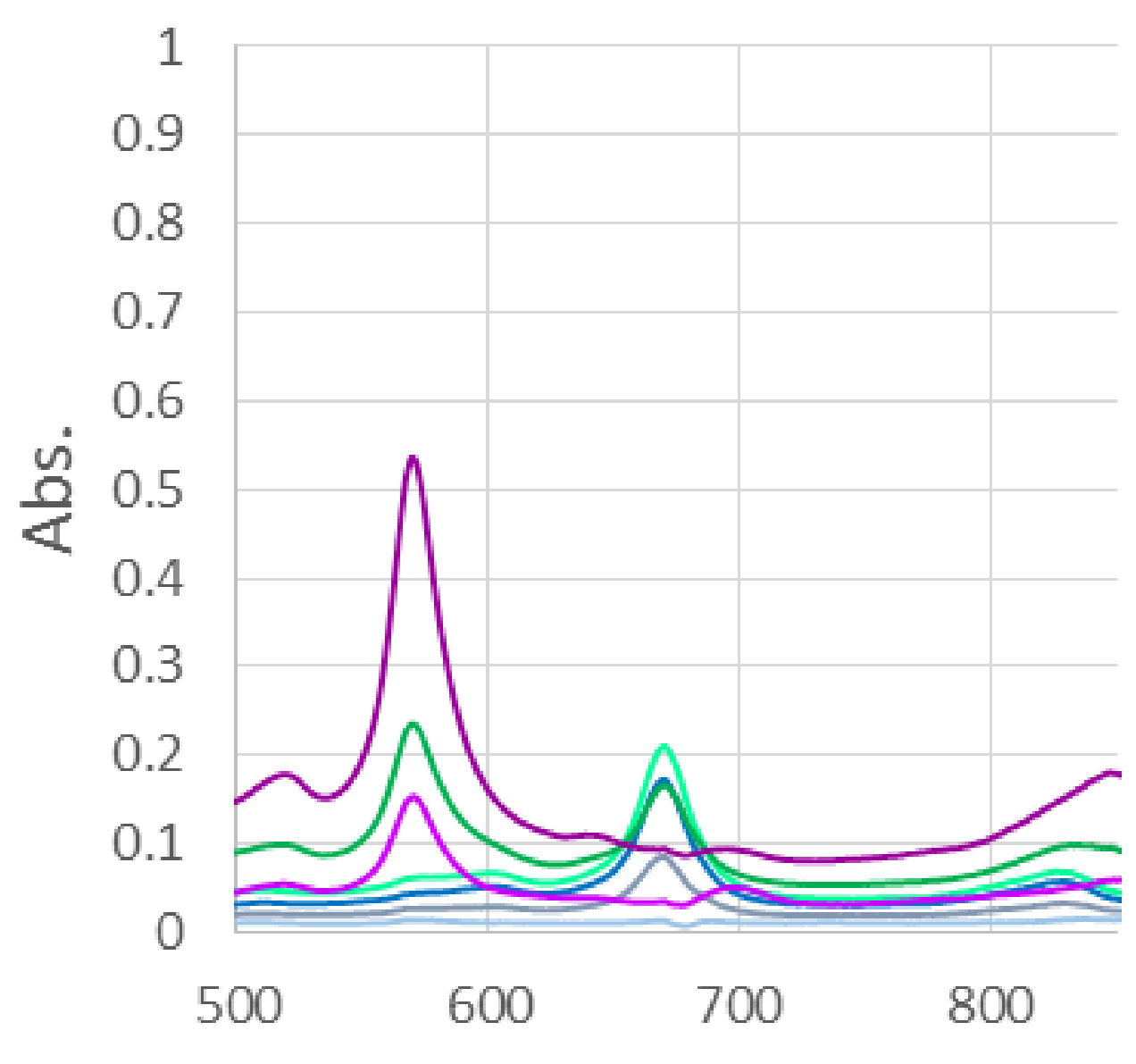

-Abs. 54 very light blue

_ Abs. 55 light blue

— Abs. 56 blue

Abs. 57 blue-green

— Abs. 58 green

— Abs. 59 purple Abs. 60 violet 


\section{spccc Carbon Nanotubes (Cheap Tubes) [04-27-16]}

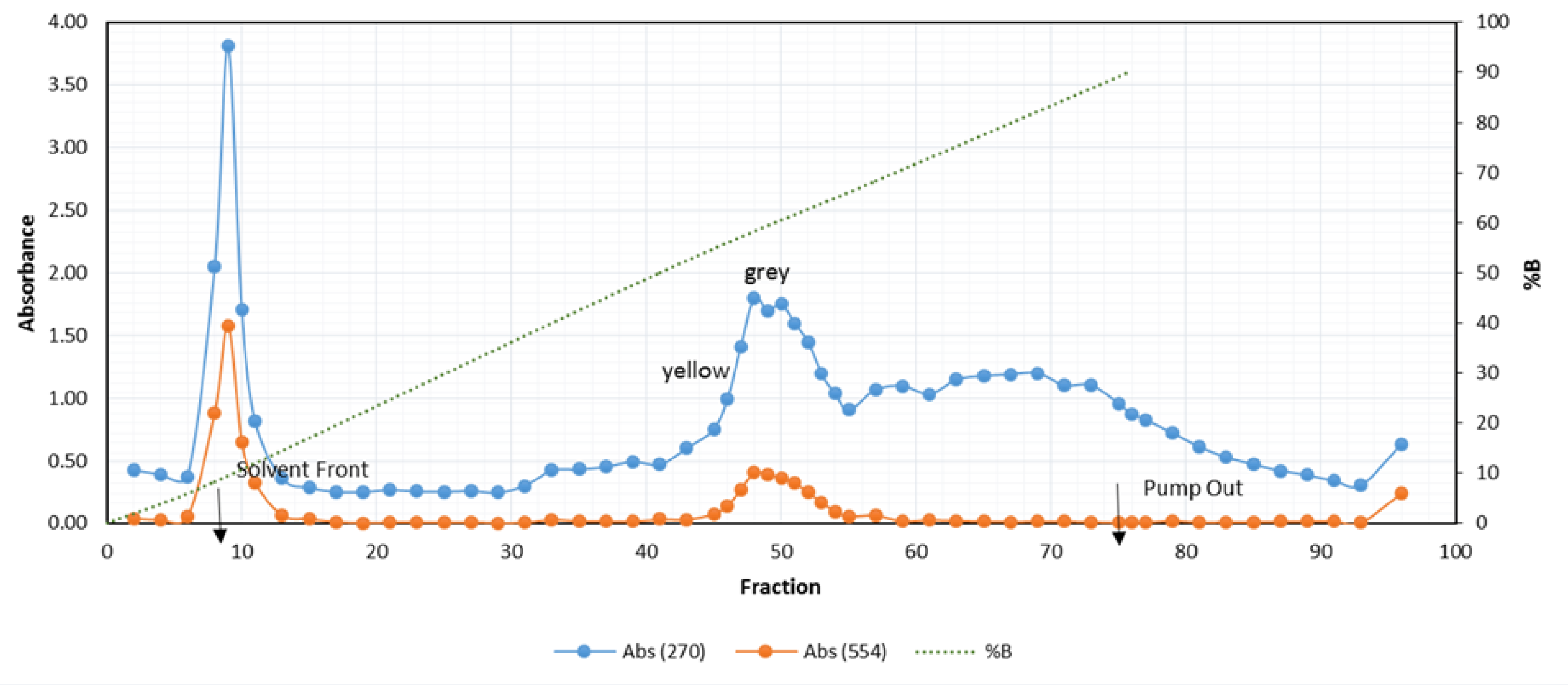

\section{Figure 8}




\section{SpCCC Carbon Nanotubes (Nano-C 0.3mg) [05/20/16]}

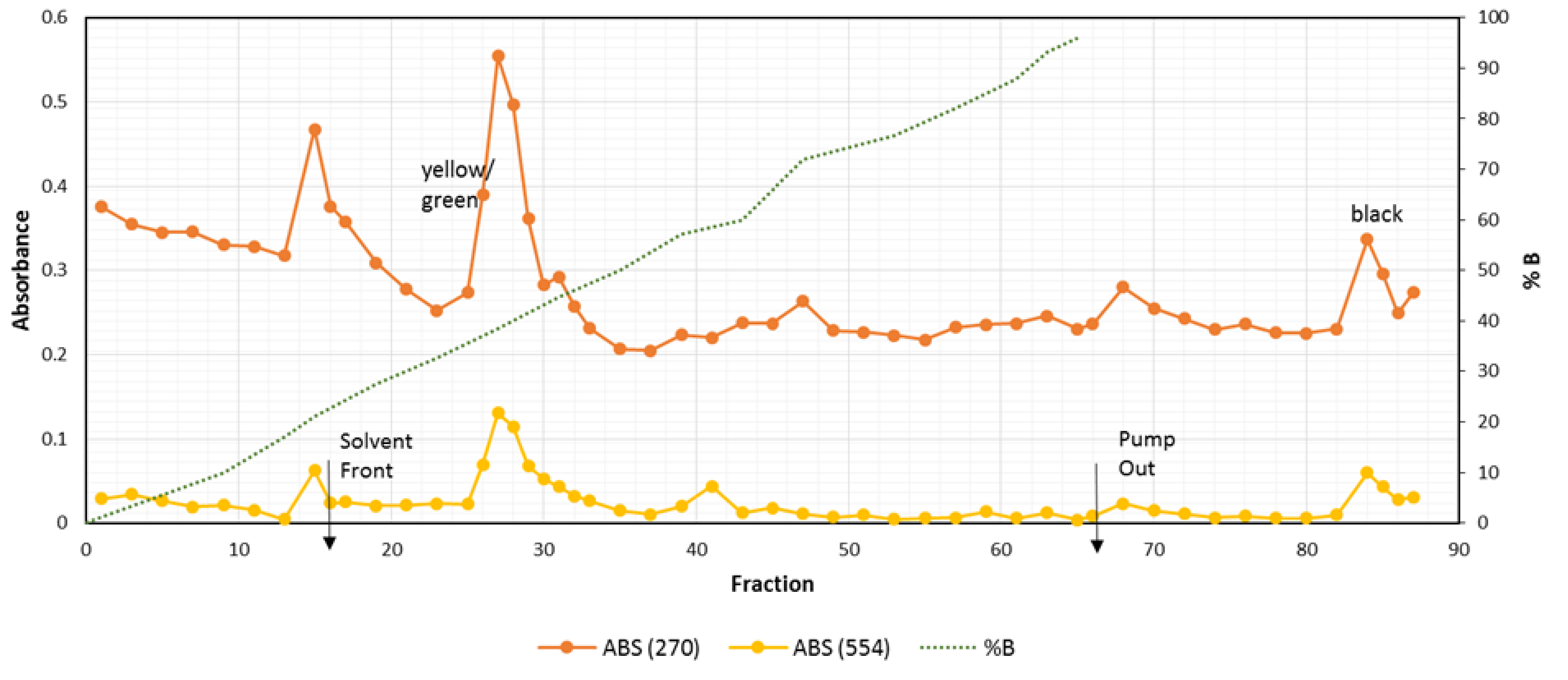

\section{Figure 9}


Table 1

\begin{tabular}{|c|c|c|}
\hline \multicolumn{3}{|c|}{ Preparation of the solvent systems for gradient spiral } \\
countercurrent chromatography \\
\hline $\begin{array}{c}\text { Stock solutions } \\
\text { By weight g/g water }\end{array}$ & $\begin{array}{c}\text { Solvent system \#1 } \\
\text { (SS\#1) }\end{array}$ & SS\#2 \\
\hline PEG 10\% & $300 \mathrm{ml}$ & $300 \mathrm{ml}$ \\
\hline DEX 16\% & $200 \mathrm{ml}$ & $200 \mathrm{ml}$ \\
\hline SDC 10\% & $1 \mathrm{ml}$ & $1 \mathrm{ml}$ \\
\hline SDS & & $3.5 \mathrm{~g}$ \\
\hline
\end{tabular}


Table 2

\begin{tabular}{|c|c|c|}
\hline $\begin{array}{l}\text { Stationary phase }=\text { LP SS\#1 } \\
\text { Mobile phase A }=\text { UP SS\#1 } \\
\text { Rotor }\end{array}$ & $\begin{array}{l}\text { itions for CNT sepa } \\
\text { UP SS\#2 } \\
\text { Conditions }\end{array}$ & er phase system \\
\hline 5 layer mixer-settler spiral disk & 0 to $100 \% \mathrm{~B}$ & 480 min linear gradient \\
\hline 7 layer mixer-settler spiral disk & 0 to $100 \% \mathrm{~B}$ & 660 min linear gradient \\
\hline $\begin{array}{l}6 \text { layer mixer-settler spiral disk } \\
\text { Mixer-settler rotor } v .2\end{array}$ & 0 to $100 \% \mathrm{~B}$ & 540 min linear gradient \\
\hline 7 layer mixer-settler spiral disk & $\begin{array}{cc}0 \text { min } & 0 \% \text { B } \\
220 \min & 50 \% \text { B } \\
440 \min & 80 \% \text { B } \\
660 \min & 100 \% \text { B }\end{array}$ & convex gradient \\
\hline
\end{tabular}


Table 3

\begin{tabular}{|l|l|l|l|l|}
\hline \multicolumn{5}{|l|}{ Solvents for gradient CCC and for sample prep } \\
\hline $\begin{array}{l}\text { Stock solutions } \\
\text { By weight g/g }\end{array}$ & $\begin{array}{l}\text { Solvent system \#1 } \\
\text { (SS\#1) }\end{array}$ & \multicolumn{3}{l|}{$\begin{array}{l}\text { Sample pre-load } \\
\text { extraction } \\
\text { SS\#3 }\end{array}$} \\
\hline PEG 10\% & $300 \mathrm{ml}$ & $4 \mathrm{ml}$ & \\
\hline DEX 16\% & $200 \mathrm{ml}$ & $300 \mathrm{ml}$ & $2.5 \mathrm{ml}$ & $2.5 \mathrm{ml}$ \\
\hline SDC $10 \%$ & $1 \mathrm{ml}$ & $200 \mathrm{ml}$ & & \\
\hline SDS & & $1 \mathrm{ml}$ & & \\
\hline PEG 14\% & & $3.5 \mathrm{~g}$ & & $4 \mathrm{ml}$ \\
\hline
\end{tabular}


Table 4

Biosensor responsiveness of CNT fractions isolated by CCC.

Starting material had the responsiveness of 1.1. ND $=$ no active circuits detected.

\begin{tabular}{|c|c|c|c|}
\hline $\begin{array}{c}\text { Fraction } \\
\text { Number }\end{array}$ & $\begin{array}{c}\text { Normalized } \\
\text { Impedance }\end{array}$ & $\begin{array}{c}\text { Absorbance } \\
(\mathbf{2 8 5} \mathbf{~} \mathbf{m})\end{array}$ & Impedance/Abs. \\
\hline 9 & 0.9 & 2.851 & 0.31 \\
\hline 10 & 1.2 & 0.992 & 1.21 \\
\hline 11 & 0.8 & 0.691 & 1.16 \\
\hline 12 & 0.9 & 0.313 & 2.88 \\
\hline 13 & ND & 0.343 & \\
\hline 48 & ND & 0.194 & \\
\hline 49 & ND & 0.206 & \\
\hline 55 & 1.57 & 0.290 & 5.41 \\
\hline 56 & 1.97 & 0.296 & 6.42 \\
\hline 57 & 2.26 & 0.323 & 7.0 \\
\hline 58 & 2.05 & 0.460 & 4.46 \\
\hline 59 & 1.16 & 0.557 & 2.08 \\
\hline 60 & 0.95 & 0.390 & 2.44 \\
\hline 62 & ND & 0.335 & \\
\hline 63 & ND & 0.355 & \\
\hline 64 & ND & 0.296 & \\
\hline
\end{tabular}

